\title{
Association between serum HER2/ErbB2 levels and coronary artery disease: a case- control study
}

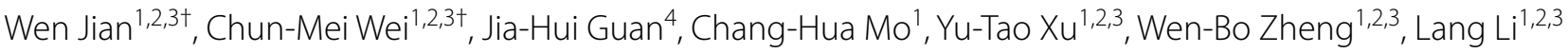 \\ and Chun Gui ${ }^{1,2,3^{*}}$ (1)
}

\begin{abstract}
Background: Research has associated human epidermal growth factor receptor (HER2) with glucose and lipid metabolism. However, the association between circulating HER2 levels and coronary artery disease (CAD) remains to be elucidated.

Methods: We performed a case-control study with 435 participants (237 CAD patients and 198 controls) who underwent diagnostic coronary angiography from September 2018 to October 2019. Adjusted odds ratios (ORs) and 95\% confidence intervals (Cls) for CAD were calculated with multiple logistic regression models after adjustment for confounders.

Results: Overall, increased serum HER2 levels were independently associated with the presence of CAD (OR per 1-standard deviation (SD) increase: 1.438, 95\% Cl 1.13-1.83; $\mathrm{P}=0.003$ ) and the number of stenotic vessels (OR per 1-SD increase: $1.399,95 \% \mathrm{Cl} 1.15-1.71 ; \mathrm{P}=0.001)$. In the subgroup analysis, a significant interaction of HER2 with body mass index (BMI) on the presence of CAD was observed (adjusted interaction $P=0.046$ ). Increased serum HER2 levels were strongly associated with the presence of CAD in participants with $B M l \geq 25 \mathrm{~kg} / \mathrm{m}^{2}$ (OR per 1-SD increase: 2.143 , $95 \% \mathrm{Cl} 1.37-3.35 ; \mathrm{P}=0.001)$, whereas no significant association was found in participants with $\mathrm{BMl}<25 \mathrm{~kg} / \mathrm{m}^{2}(\mathrm{OR}$ per 1-SD increase: $1.225,95 \% \mathrm{Cl} 0.90-1.67 ; \mathrm{P}=0.201)$.

Conclusion: Elevated HER2 level is associated with an increased risk of CAD, particularly in people with obesity. This finding yields new insight into the pathological mechanisms underlying $C A D$, and warrants further research regarding HER2 as a preventive and therapeutic target of CAD.
\end{abstract}

Keywords: Coronary artery disease, HER2/ErbB2, Risk factor

\section{Background}

Human epidermal growth factor receptor (HER) proteins are a family of receptors with tyrosine kinase catalytic activity. The HER family consists of four members,

\footnotetext{
*Correspondence: guichun@stu.gxmu.edu.cn

${ }^{+}$Wen Jian and Chun-Mei Wei have contributed equally to this work 1 Department of Cardiology, The First Affiliated Hospital of Guangxi Medical University, 06 Shuangyong Road, Nanning 530021, Guangxi, People's Republic of China

Full list of author information is available at the end of the article
}

namely, HER1/ErbB1/EGFR, HER2/ErbB2, HER3/ ErbB3, and HER4/ErbB4. Signaling cascades initiated by these receptors can mediate cell growth, survival, and differentiation through various pathways [1]. HER2, a $185-\mathrm{kDa}$ transmembrane protein, plays a critical role in facilitating uncontrolled cell growth and tumorigenesis in various cancers $[2,3]$. HER2 comprises an extracellular domain, a transmembrane domain, and an intracellular tyrosine kinase domain. Although no specific ligand has been found, HER2 can be activated upon homo- and

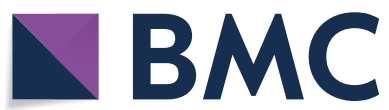

(c) The Author(s) 2020. This article is licensed under a Creative Commons Attribution 4.0 International License, which permits use, sharing, adaptation, distribution and reproduction in any medium or format, as long as you give appropriate credit to the original author(s) and the source, provide a link to the Creative Commons licence, and indicate if changes were made. The images or other third party material in this article are included in the article's Creative Commons licence, unless indicated otherwise in a credit line to the material. If material is not included in the article's Creative Commons licence and your intended use is not permitted by statutory regulation or exceeds the permitted use, you will need to obtain permission directly from the copyright holder. To view a copy of this licence, visit http://creativeco mmons.org/licenses/by/4.0/. The Creative Commons Public Domain Dedication waiver (http://creativecommons.org/publicdomain/ zero/1.0/) applies to the data made available in this article, unless otherwise stated in a credit line to the data. 
heterodimerization with another member of the HER family. As an important signal amplifier, HER2 mediates diverse functions related to the HER family [4]. Notably, HER2 signaling was found to be significantly enhanced after proteolytic cleavage of its extracellular domain (known as HER2 shedding) [5]. As the cleaved soluble HER2 proteins can be easily measured in the bloodstream, circulating HER2 levels have relevant clinical potential.

Although important to homeostasis, abnormal regulation or production of HER2 can promote diseases [2, $3]$. Intriguingly, clinical evidences have recently associated HER2 activity with glucose and lipid metabolism [6-9]. Circulating HER2 levels are found to be positively correlated with the parameters of obesity and insulin resistance [7, 8], and increased circulating HER2 levels are associated with a higher risk of developing diabetes [9]. Moreover, circulating HER2 has been identified as a causal mediator of chronic kidney disease (CKD) and can be regulated by the renin-angiotensin system [10]. These findings confirm the causal role of HER2 in various diseases besides cancer.

HER2 is abundantly expressed throughout the cardiovascular system, including by endothelial cells, vascular smooth muscle cells (SMCs), and cardiomyocytes [11-13]. Previous studies found that the HER family, particularly EGFR and HER2, contribute to the pathogenesis of vascular remodeling and atherosclerosis, such as oxidative stress, macrophage infiltration, and SMC proliferation and migration [13-16]. However, in the clinical setting, the relation between circulating HER2 levels and coronary artery disease (CAD) remains to be elucidated. With the aforementioned background, this case-control study aimed to investigate whether circulating HER2 levels are associated with CAD risk.

\section{Methods}

\section{Study population}

We selected consecutive symptomatic patients without known CAD who presented to the outpatient department and were scheduled for diagnostic coronary angiography in the First Affiliated Hospital of Guangxi Medical University, China, from September 2018 to October 2019. The following exclusion criteria were applied: (a) acute myocardial infarction, acute decompensated heart failure, tachyarrhythmia and severe comorbidities; (b) valvular or congenital heart disease, severe inflammatory conditions, autoimmune diseases, tumor, and end-stage renal disease; (c) missing samples for biomarker measurement due to numerous reasons. Based on the angiographic results recorded by experienced angiographers, patients with stenosis $\geq 50 \%$ of at least one epicardial vessel were enrolled as CAD group. In the meantime, patients without any visible lesion were chosen as control group, while those with mild coronary stenosis $(<50 \%)$ were excluded from this study. A total of 435 patients (CAD group, $n=237$; control group, $n=198$ ) were eligible and included for the final analysis. The study was approved by the Human Research Ethics Committee of the First Affiliated Hospital of Guangxi Medical University, China. All patients provided written informed consents.

\section{Data collection and related definition}

For each participant, the following baseline characteristics were collected at admission: age, gender, weight, height, smoking habit, history of hypertension, history of diabetes, history of hyperlipemia, medication history, blood pressure (systolic blood pressure, diastolic blood pressure), blood glucose (fasting glucose, hemoglobin A1c [HbA1c]), blood lipids (total cholesterol, low-density lipoprotein cholesterol [LDL-C], triglycerides, highdensity lipoprotein cholesterol [HDL-C]), and serum creatinine.

Former smoker was defined as smoking cessation for at least 6 months. Body mass index (BMI) was defined as the body mass divided by the square of the body height $\left(\mathrm{kg} / \mathrm{m}^{2}\right)$. Diabetes mellitus was defined as either a diagnosis based on the American Diabetes Association guidelines [17] or a history of taking antidiabetic medications. Hypertension was defined as the presence of systolic blood pressure $>140 \mathrm{mmHg}$, or diastolic blood pressure $>90 \mathrm{mmHg}$, or a history of taking antihypertensive medications. Dyslipidemia was defined as total cholesterol $\geq 5.2 \mathrm{mmol} / \mathrm{L}, \mathrm{LDL}-\mathrm{C} \geq 3.4 \mathrm{mmol} / \mathrm{L}$, or triglycerides $\geq 1.7 \mathrm{mmol} / \mathrm{L}$, or a history of hyperlipemia with the use of cholesterol-lowering medication. Estimated glomerular filtration rate (eGFR) was calculated with the CKD-EPI equation [18].

\section{HER2 measurement}

Overnight fasting blood samples were collected at admission for all participants. The samples were drawn into the dry tube without anticoagulant for about half an hour at room temperature, and then were processed into serum after being centrifuged at $2600 \mathrm{~g}$ for $10 \mathrm{~min}$ and stored at $-80{ }^{\circ} \mathrm{C}$. Serum HER2 levels were measured with an enzyme-linked immunosorbent assay kits (R\&D Systems, Minneapolis, MN, USA) following the manufacturer's instructions.

\section{Statistical analysis}

Continuous variables are presented as mean \pm standard deviation (SD) (for normal distributions) or median (interquartile range) (for skewed distributions), and were compared using the Student $t$ test or 
the Mann-Whitney U test, as appropriate. Categorical variables are presented as percentage of patients and compared by Chi square or Fisher exact test. Spearman correlation and multivariate linear regression model were used to test the relation between clinical variables and serum HER2 levels. Univariable and multivariable binary logistic regression were used to investigate the association of HER2 levels with the presence of CAD. Multivariable ordinal logistic regression model was established to evaluate the association of HER2 with the number of stenotic coronary vessels. Confounders were selected by clinical judgment regarding the conventional CAD risk factor. Model 1 was unadjusted. Model 2 was adjusted for age and sex. Model 3 was adjusted for age, sex, BMI, smoking habit, hypertension, diabetes, dyslipidemia, and eGFR. For each model, the $\mathrm{z}$ scores of HER2 were included as a continuous variable to calculate the odds ratios (ORs) per 1-SD increase with $95 \%$ confidence intervals (CIs). Then, we categorized all participants into quartiles according to HER2 concentrations, and calculated ORs with 95\% CIs using the lowest quartile as the reference. To assess the interaction effects of HER2 levels and conventional risk factors on the presence of CAD, interaction terms "HER $2 \times$ relevant variables" were introduced in the final multivariate model. A two-tailed $\mathrm{P}$ value $<0.05$ was regarded statistically significant. All analyses were done using SPSS, version 22.0 and MedCalc, version 18.2.1.

\section{Results}

\section{Baseline characteristics}

The clinical characteristics of the study participants are detailed in Table 1. Compared with the controls, CAD patients were older, predominantly male, and had a higher frequency of smoking, hypertension, and diabetes. In addition, CAD patients had higher levels of HbA1c, fasting glucose, triglycerides, and serum creatinine, but lower levels of HDL-C, and eGFR. No significant differences in BMI, total cholesterol, and LDL-C were found between CAD patients and controls. CAD patients had a higher frequency of dyslipidemia, although this was not statistically significant $(\mathrm{P}=0.067)$.

\section{Association between clinical variables and serum HER2 levels}

Spearman correlation analysis showed that serum HER2 levels were positively correlated with BMI $(r=0.312$, $\mathrm{P}<0.001)$, HbA1c $(\mathrm{r}=0.104, \mathrm{P}=0.036)$, total cholesterol $(\mathrm{r}=0.268, \mathrm{P}<0.001)$, triglycerides $(\mathrm{r}=0.276, \mathrm{P}<0.001)$, and LDL-C $(r=0.247, P<0.001)$, but negatively correlated with age $(r=-0.246, P<0.001)$. No correlations

Table 1 Baseline data

\begin{tabular}{|c|c|c|c|}
\hline & CAD group $(n=237)$ & Control group $(n=198)$ & $P$ value \\
\hline Age (years) & $61 \pm 10$ & $57 \pm 10$ & $<0.001$ \\
\hline Male gender & $176(74 \%)$ & $116(59 \%)$ & 0.001 \\
\hline BMI $\left(\mathrm{kg} / \mathrm{m}^{2}\right)$ & $24.48 \pm 3.31$ & $24.25 \pm 3.56$ & 0.475 \\
\hline Smoking habit & & & 0.001 \\
\hline Never smoker & $135(57 \%)$ & $146(74 \%)$ & \\
\hline Former smoker & $16(7 \%)$ & $10(5 \%)$ & \\
\hline Current smoker & $86(36 \%)$ & $42(21 \%)$ & \\
\hline Hypertension & $155(65 \%)$ & $107(54 \%)$ & 0.016 \\
\hline Diabetes & 77 (33\%) & $27(14 \%)$ & $<0.001$ \\
\hline Dyslipidemia & $159(67 \%)$ & $116(59 \%)$ & 0.067 \\
\hline$H b A 1_{c}(\%)$ & $6.1(5.7-6.8)$ & $5.9(5.6-6.2)$ & $<0.001$ \\
\hline Fasting glucose (mmol/L) & $4.9(4.5-5.8)$ & $4.7(4.3-5.1)$ & 0.001 \\
\hline Total cholesterol (mmol/L) & $4.73 \pm 1.17$ & $4.60 \pm 1.05$ & 0.259 \\
\hline Triglycerides (mmol/L) & $1.55(1.04-2.32)$ & $1.27(0.85-1.82)$ & $<0.001$ \\
\hline LDL-C (mmol/L) & $2.86 \pm 1.01$ & $2.74 \pm 0.87$ & 0.180 \\
\hline $\mathrm{HDL}-\mathrm{C}(\mathrm{mmol} / \mathrm{L})$ & $0.99(0.87-1.16)$ & $1.06(0.91-1.25)$ & 0.006 \\
\hline Serum creatinine $(\mu \mathrm{mol} / \mathrm{L})$ & $82(71-95)$ & $74(63-83)$ & $<0.001$ \\
\hline $\mathrm{eGFR}\left(\mathrm{mL} / \mathrm{min} / 1.73 \mathrm{~m}^{2}\right)$ & $84(72-95)$ & $93(83-99)$ & $<0.001$ \\
\hline HER2 (pg/mL) & $4851 \pm 1045$ & $4596 \pm 781$ & 0.004 \\
\hline
\end{tabular}

Continuous variables are presented as mean \pm SD (for normal distributions) or median (interquartile range) (for skewed distributions). Categorical variables are presented as $\mathrm{n}(\%)$

$B M I$ body mass index, eGFR estimated glomerular filtration rate, $H b A 1_{c}$ hemoglobin A1c, HDL-C high-density lipoprotein cholesterol, HER2 human epidermal growth factor receptor 2, LDL-C low-density lipoprotein cholesterol 
were found between serum HER2 levels and fasting glucose, HDL-C, and eGFR. To examine the independent determinants of HER2 variability, we performed multivariate linear regression analysis with HER2 as a dependent variable. In the entire study population, BMI presented the strongest independent association with HER2 levels (Table 2). Other independent positive determinants of HER2 variability were LDL-C and triglycerides, whereas age was negatively correlated with HER2 levels. Then, we also performed multivariate linear regression in $\mathrm{CAD}$ group and control group, respectively. Similarly, there were independent associations of BMI and triglycerides with HER2 levels in both CAD and control group (Table 2). Age and LDL-C were independently correlated with HER2 levels in control group, whereas they lost correlation in CAD group. Notably, in CAD group, hypertension was also a contributor to HER2 variability. All other tested associations were not significant (Table 2).

Association between HER2 levels and the presence of CAD Serum HER2 levels were significantly higher in CAD patients than in controls $(4851 \pm 1045$ vs. $4596 \pm 781 \mathrm{pg} /$ $\mathrm{mL}, \mathrm{P}=0.004$; Fig. 1). As shown in Table 3, each 1-SD increase in serum HER2 levels was associated with a 1.323 -fold $(\mathrm{P}=0.005)$ increased risk of $\mathrm{CAD}$ in the crude model. The risk remained strongly significant after adjustment of age and sex in model 2 (OR per 1-SD increase: $1.533,95 \% \mathrm{CI}=1.23-1.91 ; \mathrm{P}<0.001)$ and after full adjustment in model 3 (OR per 1-SD increase: 1.438, 95\% $\mathrm{CI}=1.13-1.83 ; \mathrm{P}=0.003)$. When HER2 levels were analyzed as an ordinal variable, the risk of $C A D$ was 2.365 -fold $(\mathrm{P}=0.009)$ higher in the highest quartile than that in the lowest quartile (model 3, Table 3). However, the 2nd and 3rd quartiles did not show a significantly high risk $(\mathrm{P}=0.068$ and $\mathrm{P}=0.653$, respectively).

\section{Association between HER2 levels and CAD severity}

CAD patients were categorized into single-, double-, and triple-vessel disease subgroups based on the number of stenotic vessels. Of the 237 CAD patients, 69 had singlevessel disease, 74 had double-vessel disease, and 94 had triple-vessel disease. Serum HER2 levels were positively correlated with the number of stenotic vessels (Spearman correlation, $\mathrm{r}=0.126, \mathrm{P}=0.009)$. Notably, while patients with triple-vessel disease had the highest levels of HER2, no significant difference was found between single-vessel disease and controls $(4654 \pm 868$ vs. $4596 \pm 781 \mathrm{pg} / \mathrm{mL}$, $\mathrm{P}=0.605$, Fig. 2). In addition, patients with multi-vessel disease ( $\geq 2$ vessels) had significantly higher HER2 levels than single-vessel disease ( $4932 \pm 1102$ vs. $4654 \pm 868 \mathrm{pg} /$ $\mathrm{mL}, \mathrm{P}=0.041)$. In a multivariable ordinal logistic regression model, increased serum HER2 levels were independently associated with the number of stenotic vessels (OR per 1-SD increase: $1.399,95 \%$ CI $1.15-1.71 ; \mathrm{P}=0.001$; Table 4).

\section{Interaction of HER2 with conventional risk factors on the presence of CAD}

Figure 3 shows a significant interaction of HER2 with $\mathrm{BMI}$ on the presence of CAD (adjusted interaction $P=0.046)$. After full adjustment, increased serum HER2 levels were strongly associated with the presence of CAD in participants with $\mathrm{BMI} \geq 25 \mathrm{~kg} / \mathrm{m}^{2}$ (OR per 1-SD increase: $2.143,95 \%$ CI $1.37-3.35 ; \mathrm{P}=0.001$ ), but no significant association was found in participants with

Table 2 Association between clinical variables and serum HER2 levels

\begin{tabular}{|c|c|c|c|c|c|c|c|c|c|}
\hline & \multicolumn{3}{|c|}{ Entire study population } & \multicolumn{3}{|l|}{ CAD group } & \multicolumn{3}{|c|}{ Control group } \\
\hline & $\beta$ coefficient & $95 \% \mathrm{Cl}$ & $\mathbf{P}$ & $\beta$ coefficient & $95 \% \mathrm{Cl}$ & $\mathbf{P}$ & $\beta$ coefficient & $95 \% \mathrm{Cl}$ & $\mathbf{P}$ \\
\hline Age & -0.158 & -0.272 to -0.044 & 0.007 & -0.112 & -0.298 to 0.075 & 0.240 & -0.257 & -0.395 to -0.119 & $<0.001$ \\
\hline Male gender & 0.048 & -0.171 to 0.268 & 0.665 & -0.202 & -0.559 to 0.155 & 0.265 & 0.205 & -0.059 to 0.470 & 0.127 \\
\hline BMI & 0.212 & 0.114 to 0.310 & $<0.001$ & 0.335 & 0.183 to 0.487 & $<0.001$ & 0.133 & 0.008 to 0.257 & 0.037 \\
\hline Current smoking & 0.164 & -0.059 to 0.387 & 0.149 & 0.287 & -0.032 to 0.606 & 0.078 & -0.088 & -0.392 to 0.215 & 0.567 \\
\hline Hypertension & -0.174 & -0.360 to 0.012 & 0.067 & -0.297 & -0.588 to -0.007 & 0.045 & -0.096 & -0.324 to 0.132 & 0.408 \\
\hline $\mathrm{HbA1c}$ & 0.086 & -0.005 to 0.176 & 0.064 & 0.032 & -0.090 to 0.154 & 0.607 & 0.093 & -0.053 to 0.238 & 0.210 \\
\hline LDL-C & 0.180 & 0.090 to 0.269 & $<0.001$ & 0.106 & -0.022 to 0.234 & 0.106 & 0.218 & 0.085 to 0.351 & 0.001 \\
\hline HDL-C & 0.082 & -0.010 to 0.175 & 0.080 & 0.013 & -0.106 to 0.132 & 0.835 & 0.172 & -0.007 to 0.351 & 0.060 \\
\hline Triglycerides & 0.184 & 0.092 to 0.276 & $<0.001$ & 0.175 & 0.049 to 0.301 & 0.007 & 0.197 & 0.053 to 0.341 & 0.008 \\
\hline eGFR & -0.021 & -0.126 to 0.085 & 0.697 & 0.067 & -0.081 to 0.216 & 0.372 & -0.084 & -0.235 to 0.067 & 0.271 \\
\hline
\end{tabular}

Multivariate linear regression analysis with HER2 levels as a dependent variable in the entire study population, CAD group, and control group, respectively. The $\beta$ coefficient for the continuous variables is expressed as per 1-SD increase to allow comparison among effects

$B M I$ body mass index, $C l$ confidence interval, eGFR estimated glomerular filtration rate, $H b A 1 c$ hemoglobin $A 1 C, H D L-C$ high-density lipoprotein cholesterol, $L D L-C$ lowdensity lipoprotein cholesterol 


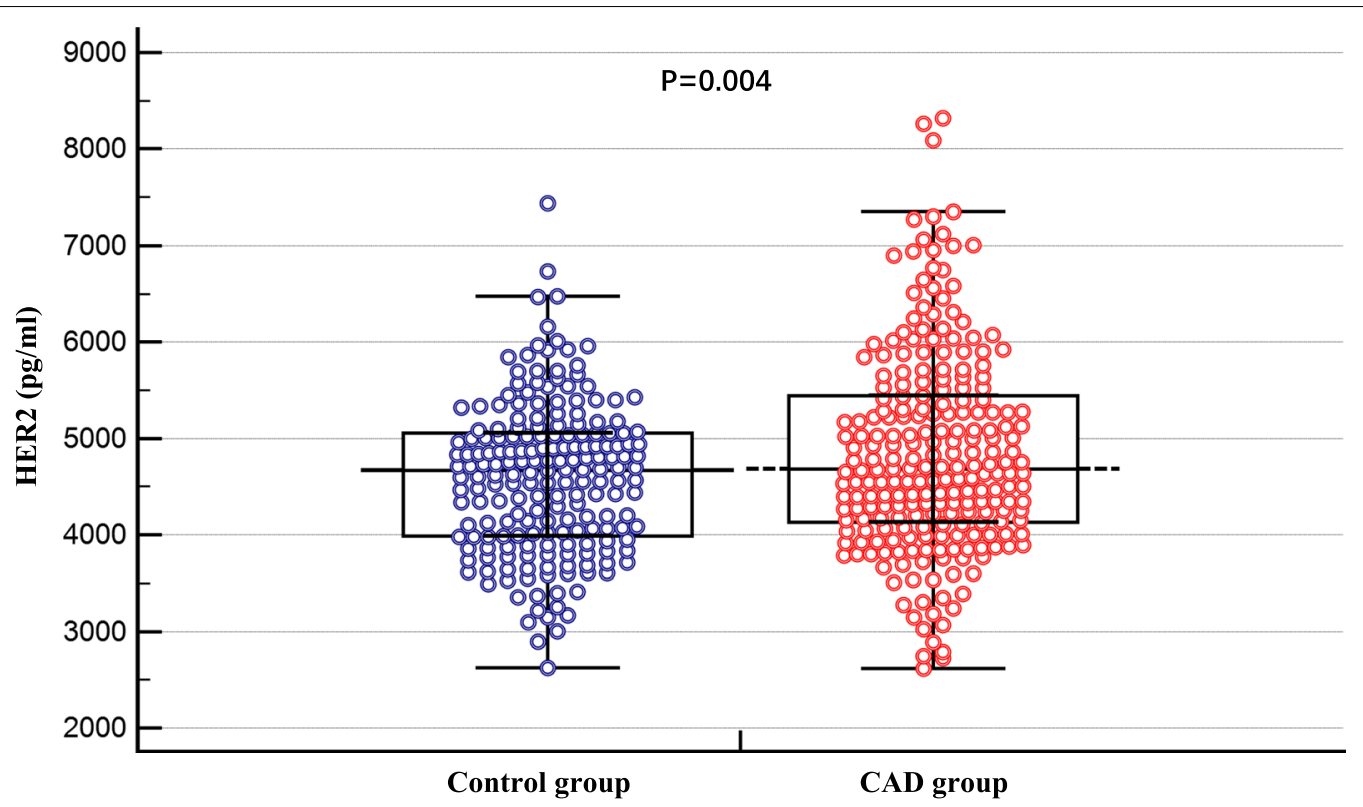

Fig. 1 Differences of the serum HER2 levels between CAD and control group

Table 3 Binary logistic regression analyses for the association of serum HER2 levels with the presence of CAD

\begin{tabular}{|c|c|c|c|c|c|c|}
\hline & \multicolumn{2}{|l|}{ Model 1} & \multicolumn{2}{|l|}{ Model 2} & \multicolumn{2}{|l|}{ Model 3} \\
\hline & OR $(95 \% \mathrm{Cl})$ & $P$ value & OR $(95 \% \mathrm{Cl})$ & $P$ value & OR (95\% Cl) & $P$ value \\
\hline Each 1-SD increase & $1.323(1.086-1.612)$ & 0.005 & $1.533(1.231-1.908)$ & $<0.001$ & $1.438(1.130-1.830)$ & 0.003 \\
\hline \multicolumn{7}{|l|}{ Quartiles of HER2 } \\
\hline Quartile 1 & 1 & - & 1 & - & 1 & - \\
\hline Quartile 2 & $1.476(0.863-2.524)$ & 0.155 & $1.578(0.892-2.791)$ & 0.117 & $1.757(0.960-3.216)$ & 0.068 \\
\hline Quartile 3 & $0.787(0.461-1.343)$ & 0.379 & $0.929(0.527-1.636)$ & 0.798 & $0.869(0.471-1.602)$ & 0.653 \\
\hline Quartile 4 & $2.104(1.215-3.644)$ & 0.008 & $2.866(1.579-5.202)$ & 0.001 & $2.365(1.242-4.503)$ & 0.009 \\
\hline
\end{tabular}

Model 1 was the unadjusted model; Model 2 was adjusted for age and sex; Model 3 was adjusted for age, sex, BMI, smoking habit, hypertension, diabetes, dyslipidemia, and eGFR. Cl, confidence interval; HER2, human epidermal growth factor receptor 2; OR, odds ratio; SD, standard deviation

$\mathrm{BMI}<25 \mathrm{~kg} / \mathrm{m}^{2}$ (OR per 1-SD increase: $1.225,95 \% \mathrm{CI}$ $0.90-1.67 ; \mathrm{P}=0.201)$. Similar results were also observed when HER2 levels were analyzed as an ordinal variable. The adjusted OR for the highest versus the lowest quartile of HER2 was 5.099 (95\% CI 1.52-17.06; $\mathrm{P}=0.008$ ) in participants with $\mathrm{BMI} \geq 25 \mathrm{~kg} / \mathrm{m}^{2}$ and was $1.731(95 \% \mathrm{CI}$ 0.74-4.05; $\mathrm{P}=0.206)$ in participants with $\mathrm{BMI}<25 \mathrm{~kg} /$ $\mathrm{m}^{2}$. However, no interaction was observed between HER2 and age $(>60, \leq 60)$, sex (male, female), current smoking, hypertension, diabetes, dyslipidemia, and eGFR (eGFR $<60, \mathrm{eGFR} \geq 60$ ) on the presence of CAD (Fig. 3).

\section{Discussion}

To the best of our knowledge, this study is the first to report that an elevated HER2 level is associated with an increased risk of CAD, particularly in people with obesity. This association remained significant even after adjusting for conventional CAD risk factors.

The HER family has been previously implicated in the pathogenesis of atherosclerosis in various animal models. EGFR, which is the most characterized member, contributes to cardiovascular disorders by causing an increase in oxidative stress, macrophage infiltration, release of proinflammatory cytokines, and transformation of SMC phenotype by a mechanism referred to as "transactivation" $[13,15]$. EGFR can be transactivated by various factors, including endothelin-1, angiotensin II, norepinephrine, prorenin, and thrombin, which interact with other cellular communication networks in the context of atherosclerotic pathologies $[19,20]$. Many experimental studies have shown that the inhibition of EGFR signaling can significantly attenuate atherosclerosis in mice models [16, $21,22]$. As a preferred heterodimeric partner, HER2 not 


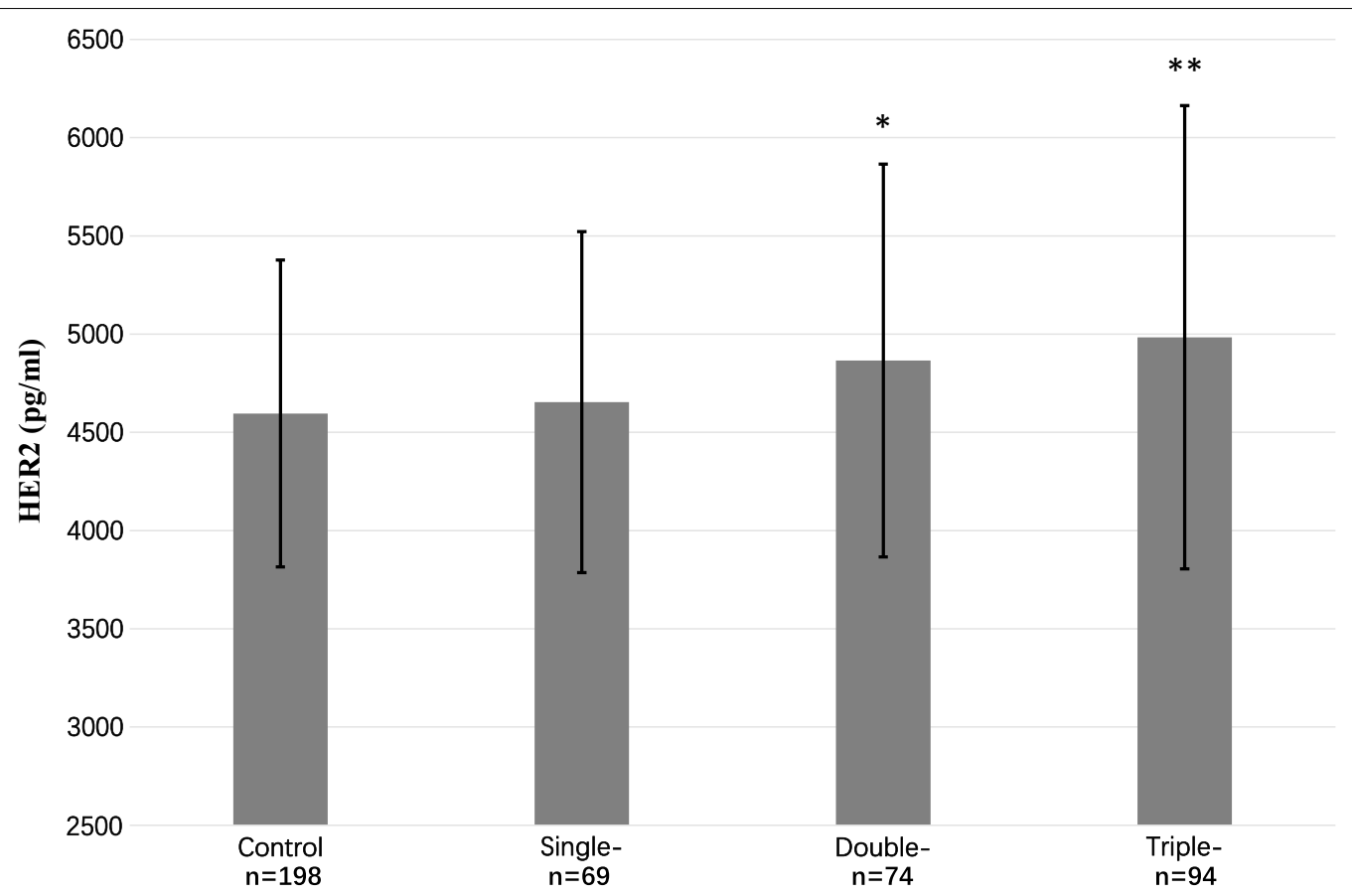

Fig. 2 Serum HER2 levels stratified by the number of stenotic vessels. Error bars represent the standard deviation of the means. ${ }^{*} \mathrm{P}<0.05$ vs Control; ${ }^{*} \mathrm{P}<0.01$ vs Control

Table 4 Ordinal logistic regression analyses for the association of clinical variables with the number of stenotic vessels

\begin{tabular}{llr}
\hline & \multicolumn{2}{l}{ Multivariate } \\
\cline { 2 - 3 } & OR (95\% CI) & P value \\
\hline Age, per 1 year & $1.046(1.022-1.070)$ & $<0.001$ \\
Male gender & $1.777(1.110-2.846)$ & 0.017 \\
BMl, per 1 kg/m² & $0.988(0.931-1.048)$ & 0.684 \\
Smoking habit & & \\
$\quad$ Former smoker & $1.303(0.589-2.886)$ & 0.513 \\
Current smoker & $2.441(1.525-3.908)$ & $<0.001$ \\
Hypertension & $1.085(0.738-1.596)$ & 0.678 \\
Diabetes & $2.580(1.670-3.988)$ & $<0.001$ \\
Dyslipidemia & $1.594(1.063-2.389)$ & 0.024 \\
eGFR, per 1 mL/min/1.73 m² & $0.983(0.971-0.996)$ & 0.011 \\
HER2, per 1 SD & $1.399(1.145-1.710)$ & 0.001 \\
\hline
\end{tabular}

The multivariable model included age, sex, BMI, smoking habit, hypertension, diabetes, dyslipidemia, eGFR, and HER2

$B M I$ body mass index, $\mathrm{Cl}$ confidence interval, eGFR estimated glomerular filtration rate, HER2 human epidermal growth factor receptor $2, O R$ odds ratio, SD standard deviation

only provides a platform which amplifies EGFR biological responses, but can also be activated through phosphorylation of its intracellular tyrosine kinase domain by EGFR; upon phosphorylation, it triggers downstream signaling
[13, 23]. Likewise, increased HER2 signaling can stimulate SMC growth, proliferation, and migration independently of EGFR downstream pathways [13]. Meanwhile, the HER2 shedding process, which is attributed to various zinc-containing metalloproteases, including matrix metallo-protease (MMP) families, can increase the tyrosine kinase activity and amplify HER2 signaling [5]. MMP families are well known to degrade structural proteins of the extracellular matrix, for example, the collagenous matrix in the fibrous cap and adjacent shoulder regions, and play critical roles in the instability of plaques [24]. Intriguingly, enhanced HER2 signaling can, in turn, promote the transcription of MMP families, thus probably creating a vicious cycle in the setting of atherosclerosis [25]. However, whether HER2 plays a role in other pathological mechanisms underlying CAD remains to be defined.

Emerging evidences suggest a close relationship between CAD and cancer, which is attributed to shared risk factors such as obesity, diabetes, hypertension, and dyslipidemia [26, 27]. However, CAD and cancer may also overlap with some potential biological pathways which have not been fully understood. Together with our findings, we assume that HER2, an oncogenic marker, is more likely to be a mediator of CAD, and people with elevated HER2 levels may possess a higher risk of CAD. Indeed, there is no evidence of reverse causation between 


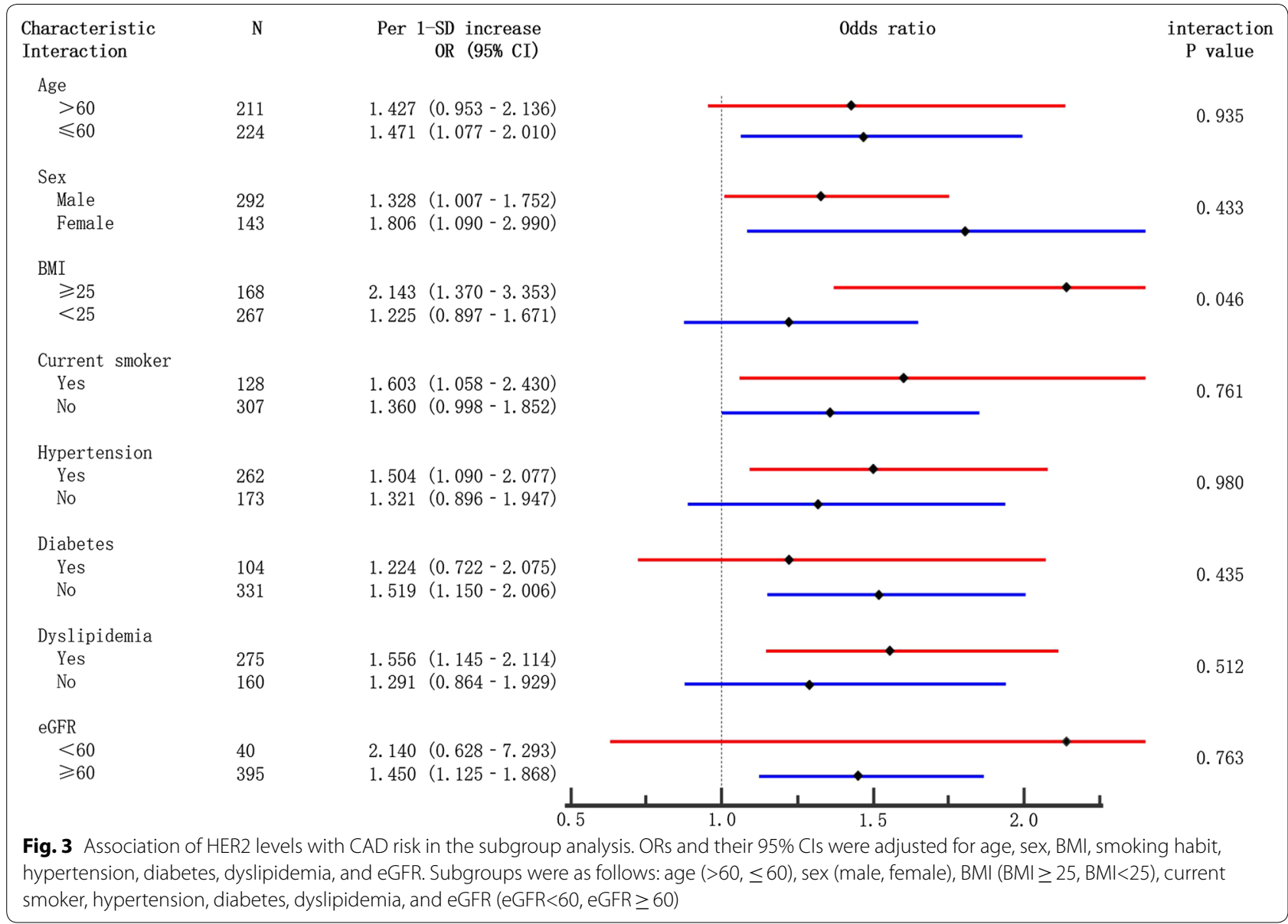

circulating HER2 levels and CAD in the current literature. In vivo, sustained hypoxia has no significant effect on HER2 expression by endothelial cells and SMCs [28, 29]. In both the early and decompensated stages of heart failure, myocardial HER2 expression has been found to be significantly decreased [30, 31]. Similarly, in biopsies from CAD patients, HER2 expression was markedly downregulated in hypoxic samples compared with that in normoxic samples [32]. Intriguingly, we found that HER2 levels in the bloodstream are higher in CAD patients than in controls, which indicates that circulating HER2 level represents the systemic activity of HER2 signaling rather than its local expression.

HER2 is essential throughout the cardiovascular system [11-13], and its extracellular domain shedding process make it highly expressed in the bloodstream [5]. Of note, our data showed that the difference in circulating HER2 levels between CAD and control groups was not strikingly huge. One possible explanation is that there is a tremendous interindividual variability in circulating HER2 levels, and it may probably have pathological relevance only when HER2 reaches a certain high level.
Muhammad et al. [9] performed a population-based cohort study, and they found that whereas participants in the highest HER2 quartile had a significant higher risk of developing diabetes than those in the lowest quartile, the 2nd and 3rd quartiles did not exhibit a higher risk after adjustment for confounders. Similarly, this phenomenon was also observed in our case-control study when we categorized all participants into quartiles according to HER2 concentrations. Given the fact that CAD is a multifactorial disease influenced by both environmental and genetic factors [33], the slightly elevated HER2 levels may not predispose people to CAD. Thus, a huge range of HER2 levels overlapped between CAD and control groups in our study. Further large cohort studies are needed to verify our findings and explore the optimal risk threshold of HER2 levels.

It is well known that patients with CKD more frequently experience cardiovascular disease than those without [34]. Considering that HER2 is a causal mediator of CKD [10], HER2 may indirectly take an influence on CAD partly through CKD. Thus, adjusting baseline eGFR in our regression models may probably underestimate the 
risk effect of HER2. Even so, we still observed an independent association between circulating HER2 levels and CAD after full adjustment in this study, which confirms a potential role for HER2 in the progress of CAD.

Obesity, which is characterized by dyslipidemia, increased blood glucose, and abnormal levels of hormone-like adipokines, mediates chronic inflammation that promotes diseases [35, 36]. MEMON et al. [8] reported that there was an independent association between HER2 levels and hyperglycemia in Swedish people. Fernandez-Real et al. [7] showed that fasting triglyceride was an independent factor which contributed to $10-11 \%$ of serum HER2 variance in a cross sectional study. They also found that serum HER2 levels could significantly decrease after weight loss in obese subjects. Similarly, in our multivariate linear regression analysis, we observed an independent association of BMI, LDL-C and triglycerides with HER2 levels, suggesting a close relation of HER2 with metabolism. Indeed, emerging evidences show that HER2 signaling is associated with obesity and the lipid-related microenvironment, and plays an important role in adipose differentiation [6,37]. Intriguingly, our study found an interaction between HER2 levels and $\mathrm{BMI}$ on the presence of $\mathrm{CAD}$, indicating that HER2 signaling may significantly contribute to the progress of atherosclerosis when coupled with other factors, such as obesity. Experimental studies found that the molecules governing lipid metabolism can significantly interact with HER2 signaling, which alters the behavior of various cell types in multiple pathological conditions [6]. For instance, leptin, an important proinflammatory adipokine, has been found to cause vascular dysfunction by increasing HER2 activity in the arterial wall [13]. In turn, HER2 signaling can enhance the transcriptional activation of leptin, which creates a feedback loop [38]. Thus, taken together, the synergistic effect of obesity and HER2 may provide new insight into the pathological mechanisms underlying CAD.

Currently, tyrosine kinase inhibitors have been widely implicated in cancers $[39,40]$. On the other hand, a growing evidence supports the notion that HER2 inhibitors are a potential treatment for diabetes [9, 41], obesity [37], and kidney disease [10, 42]. Our data suggest that HER2 may be a potential therapeutic target of CAD. However, given the possible cardiotoxicity of a potent anti-HER2 treatment that can lead to severe heart failure in breast cancer [43], the benefits and risks of this prospect warrant further study.

This study has several limitations that should be considered when interpreting its results. First, this was a single-center study with a small sample size. Only cases and controls that met the inclusion criteria were analyzed; thus, a certain degree of selection bias was present.
Given the fact that cases and controls were not age- and gender- matched, this gap was a major limiting factor for our study. Second, considering the cross-sectional nature of this study, the observational findings must be regarded as hypothesis-generating, as they do not allow for causal inference. In addition, despite the provision of plausible explanations, we could not rule out the possibility of reverse causation. Third, although conventional CAD risk factors had been adjusted, residual unknown confounders, such as unmeasured biomarkers or mechanisms, could not be entirely ruled out. Fourth, since the controls were selected from symptomatic patients with angiographically normal coronary arteries, we could not fully exclude subjects with microcirculation disturbances or angiographically invisible lesions from the control group. Thus, it remains unknown whether these conditions influence HER2 levels. More data and verification are required in the future.

\section{Conclusion}

Elevated HER2 level is associated with an increased risk of CAD, particularly in people with obesity. This finding yields new insight into the pathological mechanisms underlying $\mathrm{CAD}$, and warrants further research regarding HER2 as a preventive and therapeutic target of CAD.

\section{Abbreviations \\ BMI: Body mass index; CAD: Coronary artery disease; CKD: Chronic kidney disease; Cls: Confidence intervals; eGFR: Estimated glomerular filtration rate; HER2: Human epidermal growth factor receptor 2; HbA1c: Hemoglobin A1c; HDL-C, high-density lipoprotein cholesterol; HDL-C: High-density lipo- protein cholesterol; LDL-C: Low-density lipoprotein cholesterol; MMP: Matrix metallo-protease; ORs: Odds ratios; SD: Standard deviation; SMCs: Vascular smooth muscle cells.}

\section{Acknowledgements}

Not applicable.

\section{Authors' contributions}

CG contributed to the conception or design of the work. CG and LL contributed to the provision of resources; WJ, C-MW, J-HG, C-HM, Y-TX and W-BZ contributed to the acquisition of data. WJ and C-MW contributed to the analysis and interpretation of data. WJ was a major contributor in writing the manuscript. All authors read and approved the final manuscript.

\section{Funding}

This study was supported by the National Natural Science Foundation of China (No. 81460063), Guangxi Natural Science Foundation (No. 2014GXNSFDA118024), and the Health Technology Research and Development Project of Guangxi (No. S2019094).

\section{Availability of data and materials}

The datasets used and/or analysed during the current study are available from the corresponding author on reasonable request.

\section{Ethics approval and consent to participate}

The study has been conducted under the approval of the Human Research Ethics Committee of the First Affiliated Hospital of Guangxi Medical University,

China, and the procedures have been performed in accordance with the 
Declaration of Helsinki. Written informed consent to participate in the study was obtained from all patients enrolled in the study.

\section{Consent for publication}

Not applicable.

\section{Competing interests}

The authors declare that they have no competing interests.

\section{Author details}

${ }^{1}$ Department of Cardiology, The First Affiliated Hospital of Guangxi Medical University, 06 Shuangyong Road, Nanning 530021, Guangxi, People's Republic of China. ${ }^{2}$ Guangxi Key Laboratory Base of Precision Medicine in CardioCerebrovascular Diseases Control and Prevention, Nanning 530021, Guangxi, People's Republic of China. ${ }^{3}$ Guangxi Clinical Research Center for Cardio-Cerebrovascular Diseases, Nanning 530021, Guangxi, People's Republic of China. ${ }^{4}$ Department of Respiratory Medicine, The First Affiliated Hospital of Guangxi Medical University, Nanning 530021, Guangxi, People's Republic of China.

\section{Received: 14 January 2020 Accepted: 5 March 2020}

\section{Published online: 11 March 2020}

\section{References}

1. Wang M, Hu Y, Yu T, Ma X, Wei X, Wei Y. Pan-HER-targeted approach for cancer therapy: mechanisms, recent advances and clinical prospect. Cancer Lett. 2018:439:113-30.

2. Meric-Bernstam F, Johnson AM, Dumbrava EEl, Raghav K, Balaji K, Bhatt M, Murthy RK, Rodon J, Piha-Paul SA. Advances in HER2-targeted therapy: novel agents and opportunities beyond breast and gastric cancer. Clin Cancer Res. 2019;25:2033-41.

3. Pernas S, Tolaney SM. HER2-positive breast cancer: new therapeutic frontiers and overcoming resistance. Ther Adv Med Oncol. 2019;11:1758835919833519.

4. Schlessinger J. Ligand-induced, receptor-mediated dimerization and activation of EGF receptor. Cell. 2002;110:669-72.

5. Tse C, Gauchez AS, Jacot W, Lamy PJ. HER2 shedding and serum HER2 extracellular domain: biology and clinical utility in breast cancer. Cancer Treat Rev. 2012;38:133-42.

6. Ray A. Tumor-linked HER2 expression: association with obesity and lipidrelated microenvironment. Horm Mol Biol Clin Investig. 2017. https://doi. org/10.1515/hmbci-2017-0020.

7. Fernandez-Real JM, Menendez JA, Fruhbeck G, Moreno-Navarrete JM, Vazquez-Martin A, Ricart W. Serum HER-2 concentration is associated with insulin resistance and decreases after weight loss. Nutr Metab (Lond). 2010;7:14.

8. Memon AA, Bennet L, Zoller B, Wang X, Palmer K, Sundquist K, Sundquist J. Circulating human epidermal growth factor receptor 2 (HER2) is associated with hyperglycaemia and insulin resistance. J Diabetes. 2015;7:369-77.

9. Muhammad IF, Borne Y, Bao X, Melander O, Orho-Melander M, Nilsson PM, Nilsson J, Engstrom G. Circulating HER2/ErbB2 levels are associated with increased incidence of diabetes: a population-based cohort study. Diabetes Care. 2019;42:1582-8.

10. Sjaarda J, Gerstein HC, Yusuf S, Treleaven D, Walsh M, Mann JFE, Hess S, Pare G. Blood HER2 and uromodulin as causal mediators of CKD. J Am Soc Nephrol. 2018;29:1326-35.

11. Hedhli N, Kalinowski A. K SR: cardiovascular effects of neuregulin-1/ErbB signaling: role in vascular signaling and angiogenesis. Curr Pharm Des. 2014;20:4899-905.

12. Wu C, Gui C, Li L, Pang Y, Tang Z, Wei J. Expression and secretion of neuregulin-1 in cardiac microvascular endothelial cells treated with angiogenic factors. Exp Ther Med. 2018;15:3577-81.

13. Beltowski J, Jazmroz-Wisniewska A. Transactivation of ErbB receptors by leptin in the cardiovascular system: mechanisms, consequences and target for therapy. Curr Pharm Des. 2014;20:616-24.

14. Dreux AC, Lamb DJ, Modjtahedi H, Ferns GA. The epidermal growth factor receptors and their family of ligands: their putative role in atherogenesis. Atherosclerosis. 2006;186:38-53.
15. Schreier B, Gekle M, Grossmann C. Role of epidermal growth factor receptor in vascular structure and function. Curr Opin Nephrol Hypertens. 2014;23:113-21.

16. Wang L, Huang Z, Huang W, Chen X, Shan P, Zhong P, Khan Z, Wang J, Fang $Q$, Liang G, Wang Y. Inhibition of epidermal growth factor receptor attenuates atherosclerosis via decreasing inflammation and oxidative stress. Sci Rep. 2017;8:45917.

17. American Diabetes Association. Classification and diagnosis of diabetes: standards of medical care in diabetes-2020. Diabetes Care. 2020;43:S14-31.

18. Levey AS, Stevens LA, Schmid CH, Zhang YL, Castro AF 3rd, Feldman HI, Kusek JW, Eggers P, Van Lente F, Greene T, Coresh J. A new equation to estimate glomerular filtration rate. Ann Intern Med. 2009;150:604-12.

19. Forrester SJ, Kawai T, O'Brien S, Thomas W, Harris RC, Eguchi S. Epidermal growth factor receptor transactivation: mechanisms, pathophysiology, and potential therapies in the cardiovascular system. Annu Rev Pharmacol Toxicol. 2016;56:627-53.

20. Sanchez-Guerrero E, Jo SR, Chong BH, Khachigian LM. EGFR and the complexity of receptor crosstalk in the cardiovascular system. Curr Mol Med. 2013;13:3-12

21. Zeboudj L, Maitre M, Guyonnet L, Laurans L, Joffre J, Lemarie J, Bourcier S, Nour-Eldine W, Guerin C, Friard J, et al. Selective EGF-receptor inhibition in CD4(+) T cells induces anergy and limits atherosclerosis. J Am Coll Cardiol. 2018;71:160-72.

22. Zeboudj L, Giraud A, Guyonnet L, Zhang Y, Laurans L, Esposito B, Vilar J, Chipont A, Papac-Milicevic N, Binder CJ, et al. Selective EGFR (epidermal growth factor receptor) deletion in myeloid cells limits atherosclerosis-brief report. Arterioscler Thromb Vasc Biol. 2018;38:114-9.

23. Pagano $\mathrm{E}$, Coso $\mathrm{O}$, Calvo JC. Down-modulation of erbB2 activity is necessary but not enough in the differentiation of 3T3-L1 preadipocytes. J Cell Biochem. 2008;104:274-85.

24. Myasoedova VA, Chistiakov DA, Grechko AV, Orekhov AN. Matrix metalloproteinases in pro-atherosclerotic arterial remodeling. J Mol Cell Cardiol. 2018;123:159-67.

25. Bao W, Fu HJ, Jia LT, Zhang Y, Li W, Jin BQ, Yao LB, Chen SY, Yang AG. HER2-mediated upregulation of MMP-1 is involved in gastric cancer cell invasion. Arch Biochem Biophys. 2010;499:49-55.

26. Koene RJ, Prizment AE, Blaes A, Konety SH. Shared Risk Factors In Cardiovascular Disease And Cancer. Circulation. 2016;133:1104-14.

27. Niederseer D, Stadlmayr A, Huber-Schonauer U, Ploderl M, Schmied C, Lederer D, Patsch W, Aigner E, Datz C. Cardiovascular risk and known coronary artery disease are associated with colorectal adenoma and advanced neoplasia. J Am Coll Cardiol. 2017;69:2348-50.

28. Wang J, Zhou J, Wang Y, Yang C, Fu M, Zhang J, Han X, Li Z, Hu K, Ge J. Qiliqiangxin protects against anoxic injury in cardiac microvascular endothelial cells via NRG-1/ErbB-PI3K/Akt/mTOR pathway. J Cell Mol Med. 2017;21:1905-14

29. Kyotani Y, Ota H, Itaya-Hironaka A, Yamauchi A, Sakuramoto-Tsuchida S, Zhao J, Ozawa K, Nagayama K, Ito S, Takasawa S, et al. Intermittent hypoxia induces the proliferation of rat vascular smooth muscle cell with the increases in epidermal growth factor family and erbB2 receptor. Exp Cell Res. 2013;319:3042-50.

30. Rohrbach S, Yan X, Weinberg EO, Hasan F, Bartunek J, Marchionni MA, Lorell $\mathrm{BH}$. Neuregulin in cardiac hypertrophy in rats with aortic stenosis Differential expression of erbB2 and erbB4 receptors. Circulation. 1999;100:407-12.

31. Rohrbach S, Niemann B, Silber RE, Holtz J. Neuregulin receptors erbB2 and erbB4 in failing human myocardium-depressed expression and attenuated activation. Basic Res Cardiol. 2005;100:240-9.

32. Munk M, Memon AA, Goetze JP, Nielsen LB, Nexo E, Sorensen BS. Hypoxia changes the expression of the epidermal growth factor (EGF) system in human hearts and cultured cardiomyocytes. PLoS ONE. 2012; 7:e40243

33. Whayne TF Jr, Saha SP. Genetic risk, adherence to a healthy lifestyle, and ischemic heart disease. Curr Cardiol Rep. 2019;21:1.

34. Fujii H, Kono K, Nishi S. Characteristics of coronary artery disease in chronic kidney disease. Clin Exp Nephrol. 2019;23:725-32.

35. Piche ME, Poirier P, Lemieux I, Despres JP. Overview of Epidemiology and contribution of obesity and body fat distribution to cardiovascular disease: an update. Prog Cardiovasc Dis. 2018;61:103-13. 
36. Khan S, Shukla S, Sinha S, Meeran SM. Role of adipokines and cytokines in obesity-associated breast cancer: therapeutic targets. Cytokine Growth Factor Rev. 2013;24:503-13.

37. Vazquez-Martin A, Ortega-Delgado FJ, Fernandez-Real JM, Menendez JA. The tyrosine kinase receptor HER2 (erbB-2): from oncogenesis to adipogenesis. J Cell Biochem. 2008;105:1147-52.

38. Cha Y, Kang Y, Moon A. HER2 induces expression of leptin in human breast epithelial cells. BMB Rep. 2012;45:719-23.

39. Duchnowska R, Loibl S, Jassem J. Tyrosine kinase inhibitors for brain metastases in HER2-positive breast cancer. Cancer Treat Rev. 2018;67:71-7.

40. Jiao Q, Bi L, Ren Y, Song S, Wang Q, Wang YS. Advances in studies of tyrosine kinase inhibitors and their acquired resistance. Mol Cancer. 2018;17:36
41. Prada PO, Saad MJ. Tyrosine kinase inhibitors as novel drugs for the treatment of diabetes. Expert Opin Investig Drugs. 2013;22:751-63.

42. Melenhorst WB, Mulder GM, Xi Q, Hoenderop JG, Kimura K, Eguchi S, van Goor H. Epidermal growth factor receptor signaling in the kidney: key roles in physiology and disease. Hypertension. 2008;52:987-93.

43. Jerusalem G, Lancellotti P, Kim SB. HER2 + breast cancer treatment and cardiotoxicity: monitoring and management. Breast Cancer Res Treat. 2019;177:237-50.

\section{Publisher's Note}

Springer Nature remains neutral with regard to jurisdictional claims in published maps and institutional affiliations.
Ready to submit your research? Choose BMC and benefit from:

- fast, convenient online submission

- thorough peer review by experienced researchers in your field

- rapid publication on acceptance

- support for research data, including large and complex data types

- gold Open Access which fosters wider collaboration and increased citations

- maximum visibility for your research: over 100M website views per year

At BMC, research is always in progress.

Learn more biomedcentral.com/submissions 